\title{
Stress Imaging Alumina Corrosion Scales Using Piezofluorescence Microspectroscopy
}

\author{
D. J. Gardiner, M. Bowden and P. K. Datta \\ School of Applied Sciences, Northumbria University, Ellison Place, Newcastle upon Tyne, UK, \\ NE1 8ST
}

The stress response of the fluorescence doublet of ruby at 692.9 and $694.3 \mathrm{~nm}$ is well established and has been used as an internal pressure monitor in diamond anvil cell measurements for many years [1]. Ruby results from substitution of $\mathrm{Al}^{3+}$ by $\mathrm{Cr}^{3+}$ in $\alpha-\mathrm{Al}_{2} \mathrm{O}_{3}$ at low concentrations. The use of fluorescence piezospectroscopy has been used to monitor the stress evolution in $\alpha-\mathrm{Al}_{2} \mathrm{O}_{3}$ scales on $\mathrm{NiAl}$ single crystal (111) surfaces as a function of oxidation time using the diffusion of trace $\mathrm{Cr}^{3+}$ ions into the oxide scale to form fluorescence centres [2]. This work used an optical microprobe making single point measurements to reveal the presence of large stress gradients across and through the thickness of the scale. An unexpected broadening of the fluorescence spectra was also noted which was unexplained though thought to be due to large strain gradients within the probed volume. A contour map of the ruby fluorescence intensity of the same sample oxidised for 1 hour at $1100^{\circ} \mathrm{C}$, showing $\alpha-\mathrm{Al}_{2} \mathrm{O}_{3}$ islands has also been reported [3]. Similar measurements have been reported for $\alpha-\mathrm{Al}_{2} \mathrm{O}_{3}$ scales formed on Fe-18Cr-10Al alloy at various temperatures, using a spectroscopic arrangement which allowed both fluorescence and Raman measurements to be made at points on the surface [4]. More recently this latter group of researchers has extended the piezospectroscopic technique to the study of convoluted scales of FeCrAl alloys [5]. At this time there is no clear explanation of the unexpected bandwidth changes and preliminary measurements in our laboratory suggest that use of the fluorescence band envelope maximum as an indicator of stress may be a too simplistic approach. In addition, no attempt to determine stress images of $\alpha-\mathrm{Al}_{2} \mathrm{O}_{3}$ scales has been reported.

The unique imaging ability of the Microline Focus Spectrometer (MiFS) instrument developed at Northumbria, working in both Raman and fluorescent modes is being used to tackle some of the remaining questions of the earlier work and to extend the methodology. The MiFS instrument uses cylindrical optics to produce a line focus of an incident $\mathrm{Ar}^{+}$laser beam on the surface of the sample. Light scattered from this line is then imaged through a spectrograph to form a spectrally dispersed image on a cooled CCD detector. The non-astigmatic imaging optics employed ensure that the spatial integrity of the origin of the scattered light is retained thus generating spectral data from the length of the focused laser line at a single exposure. Raman images are obtained by collecting data from a series of adjacent lines on the sample surface.

The image shown in Figure 1 was obtained from a $100 \times 75 \mu \mathrm{m}$ area of a Co- $20 \mathrm{wt} \% \mathrm{Cr}-5 \mathrm{wt} \% \mathrm{Al}$ alloy oxidised in air at $900^{\circ} \mathrm{C}$ for 100 hours. The image was generated using the integrated intensity of the ruby fluorescence band at each of the 4800 pixel positions on the surface. The white areas are regions of high ruby concentration. Spectra obtained from points $a$ and $b$ have essentially the same bandshape and can reliably be used to determine a stress difference of $\sim 1.3 \mathrm{GPa}$ using the relationship established experimentally: $\sigma_{h}=0.133 \Delta v \mathrm{GPa}$; where $\sigma_{\boldsymbol{h}}=$ hydrostatic (isotropic) 
stress and $\Delta v=$ shift of the fluorescence signal in $\mathrm{cm}^{-1}$. Based upon an isotropic polycrystalline approximation, the in plane strain can be estimated as $3.4 \times 10^{-3}$. Stress or strain images can be rapidly generated using the shift in area centre of the fluorescence band assuming no change in bandshape.

Spectra from points $\mathrm{c}$ and $\mathrm{d}$ however have very different bandshapes which poses the question of whether the spectral shift can be used directly to assess stress. Work is under way to study corrosion scales in cross section at high spatial resolution and to explore the effects of indenter induced mechanical stress on scale integrity.
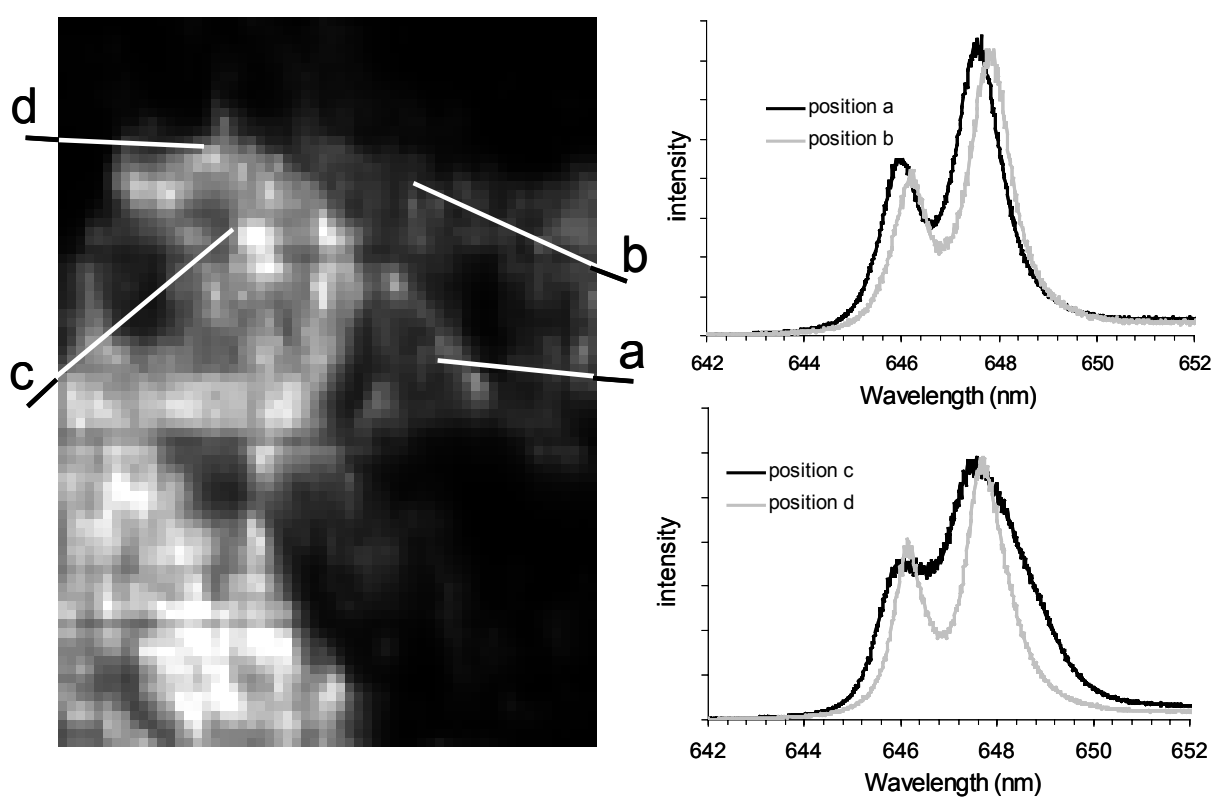

FIGURE 1. Fluorescence intensity image of oxidised CoCrAl alloy surface and indicative spectra.

\section{References}

[1] Piemarini G J and Block S, Rev. Sci. Instrum. 46, 973, (1975).

[2] D M Lipkin, D R Clarke, M Hollatz, M Bobeth and W. Pompe, Corrosion Science, 39, 231, (1997).

[3] D M Lipkin, H Schaffer, F Adar and D R Clarke, Appl. Phys. Lett., 70, 2550, (1997).

[4] D Renusch, M Grimsditch, I Koshelev, B W Veal and P Y Hou, Oxid. Met. 48, 471, (1997).

[5] J K Wright, R L Williamson, D Renusch, B Veal, M Grimsditch, Py Hou and R M Cannon, Mat.

Sci. and Eng. A262, 246, (1999). 